\title{
Vorläufiges Verzeichnis der wichtigsten Abkürzungen
}

Aarne Der reiche Mann $=F F C .23$.

A arne FinnVar.: A. Aarne, Finnische Märchenvarianten $=F F C$. 5. Hamina r9r r.

A arne Leitfaden: A. Aarne, Leitfaden der vergleichenden Märchenforschung. Hamina 1913 = FFC. 13 .

A arne Märchenliteratur: A. Aarne, Utbersicht der Märchenliteratur. Hamina I914 $_{4}=$ FFC. 14.

A arne Märchentypen: A. Aarne, Verzeichnis der Märchentypen. Helsingfors rgro $=F F C$. 3 .

A arne-Thompson: Stith Thompson, The Types of the Falk-Tale. A Classification and Bibliography. Antti Aarnes Verzeichnis der Märchentypen translated and enlarged $=$ FFC. 74. Helsinki r928.

Aarne Tiere: A. Aarne, Die Tiere auf der Wanderschaft. Hamina $19 \mathbf{r}_{3}=F F C$. Ir .

Aberglauben $W B$.: Handwörterbücher zur deutschen Volkskunde. Abt. I. Aberglaube. Herausgegeben von H. Bächtold-Stäubli. Berlin, Walter de Gruyter. Im Erscheinen.

Afanasjev: A. N. Afanasjev, Narodnyja russkija skazki. I-VIII. Moskau 1855 bis 1863. 3. Aufl. I-II, hrg. von Gruzinskij. Moskau 1897.

A ichele Zigeuner $M$. K. Block und J. Aichele, Zigeunermärchen $=M d W$., Jena, Diederichs.

Alemannia: Zeitschrift für Sprache, Literatur und Volkskunde des Elsasses und Oberrheins Iff. Bonn $1873 \mathrm{ff}$.

Aly Herodot: Wolf Aly, Volksmärchen, Sage und Novelle bei Herodot und seinen Zeit genossen. Göttingen 1921 .

ArchRelW.: Archiv für Religionswissenschaft. Iff. Freiburg i. B. $1898 \mathrm{ff}$.

Arch siebb $L k .:$ Archiv des Vereins für siebenbürgische Landeskunde.

Arch slavPhil.: Archiv für slavische Philologie. Berlin $1876 \mathrm{ff}$.

Archivio: Archivio per lo studio delle tradizioni popolari. I-XXVIII. Palermo $1882 \mathrm{ff}$.

Árnason: J. Árnason, Islenzkar pjóđsögur og Aefintýri. I-II. Leipzig 1862-1864.

Arndt Märchen: E. M. Arndt, Märchen und J ugenderinnerungen. I-II. Berlin 1818 bis 1845 .

Arnim: F. v. Arnim, Hundert neue Märchen; I. Charlottenburg 1844 .

A urbacher Büchlein: L. Aurbacher, Ein Büchlein für die Jugend. Stuttgart 1834 .

Bächtold Schweiz: H. Bächtold, Schweizer Märchen. Basel r9r6.

Bartsch Mecklenburg: K. Bartsch, Sagen, Märchen und Gebräuche aus Mecklenburg. I-II. Wien 1879 .

Bartsch Schlesien: K. Bartsch, Schlesische Märchen. Schlesische Provinzialblätter ${ }^{2}$ III-IV, Breslau I864/5.
Bechstein $D M B:$ L. Bechstein, Deutsches Märchenbuch. Leipzig 1845 .

Bechstein NDMB: L. Bechstein, Neues deutsches Märchenbuch. Wien 1856 .

Benfey Pantschatantra I. II. Leipzig 1859 .

Berendsohn Grundformen: W. A. Berendsohn, Grundformen volkstümlicher Erzählerkunst in den Kinder- und Hausmärchen der Brüder Grimm. Hamburg I921.

Berntsen: K. Berntsen, Folkeaventyr. I-II. Odense $1873-1883$. 2. Aufl. Kopenhagen I9I6.

Bethe MSM.: E. Bethe, Märchen, Sage, Mythus. Leipzig 1922.

Birlinger Schwaben: A. Birlinger, Aus Schwaben, I-II. Wiesbaden 1874 .

Birlinger Volkst.: A. Birlinger, Volkstümliches aus Schwaben. $I-2$. Freiburg i. B. 1861/2.

Bladé Gascogne: J. F. Bladé, Contes de la Gascogne. Paris 1895 .

BlPommVk.: Blätter für pommersche Volkskunde Iff. Stettin I $893 \mathrm{ff}$.

Böhm-Specht Lettische VM.: Max Böhm und F. Specht, Lettische und Litauische Märchen $=M d W$. Jena, Diederichs.

Böklen Sneewittchen: E. Böklen, Sneewittchenstudien. I-II. Leipzig I910, 1915.

Bolte Name: J. Bolte, Name und Merkmale des Märchens. Helsinki $1920=F F C$. 36 .

Bolte Zeugnisse: J. Bolte, Zeugnisse zur Geschichte der Märchen. Helsinki 192I = FFC. 39.

Bolte-Polivka: Johannes Bolte und Georg Polívka, Anmerkungen zu den Kinder- und Hausmärchen der Brüder Grimm. I-IV. Leipzig I9I3-I930.

Brunner OstdVk.: Karl Brunner, Ostdeutsche Volkskunde. Leipzig 1925.

Brueyre Grande Bretagne: L. Brueyre, Contes populaires de la Grande-Bretagne. Paris 1875 .

BSBb.: Sitzungsberichte der Berliner Akademie der Wissenschaften.

Bundi Engadin VM.: G. Bundi, Engadiner Märchen. I-II. Zürich 1902-1904.

Bundi Engadin: G. Bundi, Aus dem Engadin. Bern r913.

Bünker: J. R. Bünker, Schwänke, Sagen und Märchen in heanzischer Mundart. Leipzig 1906.

Busch: W. Busch, Ut ôler Welt. München rgro.

Campbell Clan Traditions: J. G. Campbell, Clan Traditions and Popular Tales of the Western Highlands. London 1895 .

Campbell Tales: J. F. Campbell, Popular Tales of the West-Highlands. I-IV. Edinburgh $1860-1862$. 2. Aufl. London 1890-93.

Carnoy Picardie: E. H. Carnoy, Littérature orale de la Picardie. Paris 1883 . 
Carnoy $=$ Nicolaïdes: E. H. Carnoy et $\mathrm{J}$. Nicolaïdes, Traditions populaires de I'Asie Mineure. Paris 1889.

Chalatianz: G. Chalatianz, Märchen und Sagen $=$ Armenische Bibliothek IV. Leipzig 1887 .

Chavannes RussVM.: G. Chavannes, Die russischen Volksmärchen. In: Die Wissenschaften im 19. Jh., hg. von Romberg, IX $89 \mathrm{ff}$.

Colshorn Märchen: C. u. Th. Colshorn, Märchen und Sagen. Hannover 1854 .

Comparetti: D. Comparetti, Novelline popolari italiane. Turin 1875 .

Cosquin, Lorraine: E. Cosquin, Contes populaires de Lorraine. I-II. Paris 1887 .

Cosquin Origine: E. Cosquin, Les Contes populajres et leur origine. Bruxelles 1893 .

Cowe11: E. B. Cowell, Jātaka ou Stories of the Buddha's former births. I-VI. Cambridge 1895-1907.

Curtze Waldeck: L. Curtze, Volksüberlieferungen aus dem Fürstentum Waldeck. Arolsen I860.

Dähnhardt Märchen: $O$. Dähnhardt, Deutsches Märchenbuch. I-II. Leipzig r903.

Dähnhardt Natursagen: 0 . Dähnhardt, Natursagen. I-IV. Leipzig 1907-I912.

Dähnhardt NaturVM.: O. Dähnhardt, Naturgeschichtliche Volksmärchen. Leipzig 1898 . 3. Aufl. I-II. I909.

Dardy: I. Dardy, Anthologie populaire de 1'Albret. II. Agen 1891.

Decurtins: K. Decurtins, Rätoromanische Chrestomathie II-XI. Erlangen I90I bis 1917.

Dehmer Islendinga-Sögur: Heinz Dehmer, Primitives Erzählungsgut in den fslendinga-Sögur. = Von deutscher Poeterey II. Leipzig I927.

Dirr Kaukasus: A. Dirr, Kaukasische Märchen. Jena $1920=M d W$.

Dorsey: G. A. Dorsey, Traditions of the Skidi Pawnee. Boston 1904.

Dozon: A. Dozon, Contes albanais. Paris $188 \mathrm{r}$.

Dykstra Friesland: W. Dykstra, Uit Frieslands volksleven. Leeuwarden 1894 .

Engelien-Lahn: A. Engelien und W. Lahn, Der Volksmund in der Mark Brandenburg. Berlin 1868 .

Erk-Böhme: L. Erk und F. M. Böhme, Deutscher Liederhort. I-III. Leipzig 1893-I 894.

Erman Literatur: A. Erman, Die Literatur der Ågypter. Leipzig 1923.

Erman Westcar: A. Erman, Die Märchen des Papyrus Westcar. Berlin 1890 .

Ey Harz: A. Ey, Harzmärchenbuch oder Sagen und Märchen aus dem Oberharze. Stade I862.

FFC.: F. F. Communications.

Firmenich: J. M. Firmenich, Germaniens Völkerstimmen. I-III. Berlin I 843-I 868.

Franzisci Kärnten: F. Franzisci, Märchen aus Kärnten. Klagenfurt 1884 .
Frobenius Atlantis. Volksmärchen und Volksdichtungen Afrikas I-XII. Jena r92I bis I928 (= Veröffentlichungen des Forschungsinstitutes für Kulturmorphologie).

Frommann Mundarten: G. K. Frommann, Die deutschen Mundarten. $1854 \mathrm{ff}$.

Gaal: G.v. Gaal, Märchen der Magyaren. Wien I822.

Gaster Beitr.: M. Gaster, Beiträge zur Vergleichenden Märchen- und Sagenkunde. Bukarest $188_{3}$ (= Monatsschrift für Geschichte des Judentums 29,30).

Georgeakis = Pineau: G. Georgeakis und L. Pineau, Le folk-lore de Lesbos. Paris 1894.

Gerland Odyssee: Georg Gerland, Altgriechische Märchen in der Odyssee. Magdeburg I 869.

Gerle: W. A. Gerle, Volksmärchen der Böhmen. I-II. Prag I819.

Giese Türkische Märchen: Friedrich Giese, Türkische Märchen $=M d W$. Jena, Diederichs.

Gilow Diere: Ch. Gilow, De Diere, as man to seggt un wat's seggen. 1871 .

Godin PolnVM.: A. Godin, Polnische Volksmärchen. Leipzig 1877 .

Gonzenbach: L. Gonzenbach, Sizilianische Volksmärchen. I-II. Leipzig 1870 .

Götzinger Reallexikon: E. Götzinger, Reallexikon der deutschen Altertümer. 2. Aufl. 1885 .

Goyert Vlaemische Märchen. Jena, Diederichs. 1925.

Goyert-Wolter: G. Goyert und K. Wolter, Vlaemische Sagen, Legenden und Volksmärchen. Jena r9i7.

Grimm Mythologie: Jacob Grimm, Deutsche Mythologic. 4. Ausg. Berlin $1875-1878$. I-III.

Grimm RA: Jakob Grimm, Deutsche Rechtsaltertümer 4. Ausg. I-II. Leipzig I 899.

Grünbaum Chrestomathie: M. Grünbaum, Jüdisch-deutsche Chrestomathie. Leipzig 1882 .

Grundtvig Gamle danske Minder i Folkemunde. I-III. Kopenhagen $1854-\mathrm{r} 86 \mathrm{I}$.

Gubernatis S. Stefano: A. de Gubernatis, Novelline di Santo Stefano di Calcinaja. Turin 1869 und Rom 1894 .

Gubernatis Tiere: A. de Gubernatis, Die Tiere in der indogermanischen Mythologie. Deutsch von M. Hartmann. Leipzig 1874 .

Gunkel $A T$.: H. Gunkel, Das Märchen im Alten Testament. Tübingen I92 I.

Haas Rügen: A. Haas, Rügensche Sagen und Märchen. Greifswald I891. 2. Aufl. I896, 3. Aufl. Stettin 1903.

Hackmann Katalog: O. Hackmann, Katalog der Märchen der finnländischen Schweden. Leipzig rgr I (= FFC. 6.)

Hahn Griechische M.: J. G. v. Hahn, Griechische und albanesische Märchen. I-II. Leipzig I 864 .

Haltrich VM.: J. Haltrich, Deutsche Volksmärchen aus dem Sachsenlande in Siebenbürgen. Berlin 1856. 4. Aufl. Wien 1885 . 
Hamann Vorlagen: H. Hamann, Die literarischen Vorlagen der Kinder- und Hausmärchen und ihre Bearbeitung durch die Brüder Grimm. Palaestra 47. Berlin r9o6.

Hambruch Sudsee: Paul Hambruch, Südseemärchen $=M d W$. Jena, Diederichs.

Hambruch MalM.: Paul Hambruch, Malaische Märchen $=M d W$. Jena, Diederichs.

Hartland Perseus: E. S. Hartland, The Legend of Perseus. I-III. London $1894-1896$.

Heckscher $V k$.: K. Heckscher,Volkskunde des germanischen Kulturkreises. Hamburg 1925 .

Heimat: Aus der Heimat.

Hertel Indische Märchen: J. Hertel, Indische Märchen. Jena. $=M d W$.

Hertel Pañcatantra: J. Hertel, Das Pañcatantra, seine Geschichte und seine Verbreitung. Leipzig I914.

HessBlVk.: Hessische Blätter für Volkskunde.

Hoops Reallexikon: Johannes Hoops, Reallexikon der germanischen Altertumskunde. I-IV. Straßburg I9II-rgr9.

Hovorka-Kronfeld: O. v. Hovorka und A. Kronfeld, Vergleichende Volksmedizin. I-II. Stuttgart 1908-I909.

Huet Contes populaires: Gédéon Huet, Les Contes populaires. Paris 1923.

Ilg Maltesische Mürchon: B. Ilg, Mailesische Märchen und Schwänke. I-II. Leipzig r906- 1907 .

Jahn PommernVM.: U. Jahn, Volksmärchen aus Pommern. I. Norden I89r.

J annsen $E \operatorname{stn} M .:$ H. Janusen, Märchen und Sagen des estnischen Volkes. I-II. Dorpat I881. Riga r 888 .

J anson: $K$. Janson, Folke-Eventyr uppskrivene $i$ Sandeherad. Kristiania 1878 .

Jecklin: D. Jecklin, Volkstümliches aus Graubünden. I-III. Chur I874-I 878 .

Jegerlehner Oberwallis: J. Jegerlehner, Sagen und Märchen aus dem Oberwallis. Basel r913.

Jegerlehner Sennen I: J. Jegerlehner, Was die Sennen erzählen. 3. Aufl. Bern r9o8.

Jegerlehner Sennen II: J. Jegerlehner, Am Herdfeuer der Sennen. Bern 1908.

Joos Vertelses: A. Joos, Vertelses van het vlaamsche Volk. I-IV. Brügge $1889-$ 1892 .

Jungbauer Böhmerwaldmärchen. Passau r923.

Jungbauer Turkestan: Gustav Jungbauer, Märchen aus Turkestan und Tibet $=M d W$. Jena, Diederichs.

Jurkschat: C. Jurkschat, Litauische Märchen und Erzählungeu. I. Heidelberg 1898 .

Kahlo Verse: G. Kahlo, Die Verse in den Sagen und Märchen. Diss. Jena 19r9.

Kallas: O. Kallas, Märchen der Ljutziner Esten. In: Verhandlungen der Gelehrten estnischen Gesellschaft. XX. Jurjew r9oo.

KaradżiC: Zeitschrift für serbisches Volksleben, Gebräuche und Traditionen. I-III. Alexinac r899-r9or.

KHM.: Jacob und Wilhelm Grimm, Kinderund Hausmärchen.
Kl apper Mittelalter: J. Klapper, Erzählungen des Mittelalters in deutscher Utbersetzung und lateinischem Urtext. Breslau r9r4.

Kletke Almanach: H. Kletke, Almanach deutscher Volksmärchen. Berlin I 840 .

Kletke Märchensaal. I-III. Berlin 18441845 .

Knoop Hinterpommern: O. Knoop, Volkssagen, Erzählungen, Aberglauben, Gebräuche und Märchen aus dem östlichen Hinterpommern. Posen 1885 .

Knoop Posen: O. Knoop, Posener Märchen. Programm Rogasen rgog.

Knoop Tierwelt: $\mathrm{O}$. Knoop, Volkstümliches aus der Tierwelt. Rogasen r905.

Knowles Kaschmir: J. H. Knowles, Folk-Tales of Kashmir. 2. Aufl. London 1893 .

Koch-Grünberg Indianermärchen: Th. KochGrünberg, Indianermärchen aus Südamerika. $=M d W$. Jena, Diederichs.

Köhler Aufsätze: R. Köhler, Aufsätze über Märchen und Volkslieder. Berlin r 894.

Köhler $K l S c h r .:$ R. Köhler, Kleinere Schriften, herausgegeben von J. Bolte. I-III. Weimar r 898-r 900 .

Konrad: H. Konrad, Neues Märchenbuch. 2. Aufl. Lissa 1906 .

Korr $n \boldsymbol{d}$ Sprachi.: Korrespondenzblatt des Vereins für niederdeutsche Sprachforschung. Iff. Hamburg $1877 \mathrm{ff}$.

Krainz: J. Krainz, Mythen und Sagen aus dem steirischen Hochlande. Bruck 1880 .

Krau $B S M$.: F. Krauß, Sagen und Märchen der Südslaven. I-II. Leipzig I883-1884.

KrauB rooo $S M$.: F. KrauB, Tausend Sagen und Märchen der Südslaven. I. Leipzig I9I4.

Kretschmer Neugriechisch: P. Kretschmer, Neugriechische Märchen. Jena 1917.

Kreutzwald: F. Kreutzwald, Esthnische Märchen, übersetzt von F. Löwe. I-II. Halle r869, Dorpat 1881 .

Krickeberg Indianermärchen: W. Krickeberg, Indianermärchen aus Nordamerika. Jena $1924=M d W$.

Kristensen Sagn: E. Tang Kristensen, Danske Skjaemtesagn. I. Aarhus I900.

Krohn Bär: K. Krohn, Bär (Wolf) und Fuchs. Helsingfors 1888 .

Krohn Mann: K. Krohn, Mann und Fuchs. Helsingfors $\mathbf{r} 8 \mathbf{9}$.

Krohn Methode: K. Krohn, Die folkloristische Arbeitsmethode. Oslo 1926.

Kuhn Mark: A. Kuhn, Märkische Sagen und Märchen. Berlin 1843 .

Kuhn Westfalen: A. Kuhn, Sagen, Gebräuche und Märchen aus Westfalen. I-II. Leipzig 1859 .

Kuhn-Schwartz Nordd. Sagen: A. Kuhn und W. Schwartz, Norddeutsche Sagen, Märchen und Gebräuche. Leipzig 1848 .

Laistner Sphinx: Ludwig Laistner, Das Rätsel der Sphinx. Berlin 1889.

Lederbogen: W. Lederbogen, Kameruner Märchen. Berlin I902. 
Lefèv re Perrault: André Lefèvre, Les Contes de Perrault. Paris o. J.

Lefftz Grimm: J. Lefftz, Märchen der Brüder Grimm. Urfassung. Heidelberg 1927.

L,eibing Berg: F. Leibing, Sagen und Märchen des Bergischen Landes. Elberfeld 1868 .

Lemke Ostpreußen: E. Lemke, Volkstümliches aus Ostpreußen. I-III. Mohrungen I 884 . 1887. Allenstein 1899 .

Lenz Engl Märchen: L. Lenz, Die neuesten englischen Märchensammlungen und ihre Quellen. Kassel 1902.

Liljeblad Tobias: Sven Liljeblad, Die Tobiasgeschichte und andere Märchen mit toten Helfern. L,und 1927 .

Leskien: A. Leskien, Balkanmärchen aus Albanien, Bulgarien, Serbien und Kroatien. Jena 1915.

v. d. Leyen Märchen: F. v. d. Leyen, Das Märchen. 3. Aufl. Leipzig 1925.

v. d. Leyen Eddamärchen: F. v. d. Leyen, Das Märchen in den Göttersagen der Edda. Berlin 1898 .

Liebrecht $V K$.: Felix Liebrecht, Zur Volkskunde: Heilbronn 1879 .

Littmann rOOI Nacht: Enno Littmann, roOI Nacht. Insel-Verlag, seit I923.

Löwis of Menar Finnische VM.: A. v. Löwis of Menar, Finnische und estnische Volksmärchen $=M d W$. Jena, Diederichs.

Löwis of Menar Held: A. v. Löwis of Menar, Der Held im deutschen und russischen Märchen. Jena 19r2.

Löwis of Menar RussischeVM.: A. von Löwis of Menar. Russische Volksmärchen. Jena 1914. $=M d W$.

Lüders BuddhMärchen: Else Lüders, Buddhistische Märchen aus dem alten Indien := $M d W$. Jena, Diederichs.

Luzel Rapports: F. M. Luzel, Rapports sur une mission en Basse-Bretagne. Archives des missions scientifiques et littéraires. ${ }^{2}$ VII; 3I. $1872-1873$.

Mackensen Der singende Knochen $=$ FFC. 49 .

Marianu: St. F. Marianu, Ornitologia popolană româñ̆ I-II. Cernauti 1883 .

Maspero Contes populaires: Maspero, Les contes populaires des Anciens Egyptiens. Paris o. J.

$M d W .:$ Die Märchen der Weltliteratur, hrg. von F. v. d. Leyen und Paul Zaunert. Jena, Diederichs.

Meier Schwaben.: E. Meier, Deutsche Volksmärchen aus Schwaben. Stuttgart. 1852 .

Meinhof Afrika: Karl Meinhof, Afrikanische Märchen $=M d W$. Jena, Diederichs.

Meyer AlbanMärchen: G. Meyer, Albanische Märchen. Archiv für Literaturgeschichte. XII. 1884 .

Meyer PlattdVM.: G. F. Meyer, Plattdeutsche Volksmärchen und Schwänke. Neumünster 1925.

Mijatovics: C. Mijatowics, Serbian Folk-tales, ed. by W. Denton. London 1874 .
Mikuličić: F. Mikuličić, Narodne pripovietke iz hrvatskoga primorja. Portorè 1876 .

Milenowsky Böhmen.: J. Milenowsky, Volksmärchen aus Böhmen. Breslau 1853 .

Monteil Contes soudanais. Paris 1905 .

Mont $=$ Cock: P. de Mont en A. de Cock, Vlamsche Wondersprookjes. Gent I896.

Müllenhoff Schleswig: K. Müllenhoff, Sagen, Märchen und Lieder der Herzogtümer Schleswig, Holstein und Lauenburg. Kiel 1845 .

Müller Psychologie: E. Müller, Psychologie des deutschen Volksmärchens. München 1928.

Müller Siebenbürgen: Friedrich Müller, Siebenbürgische Sagen. = Siebenbürgisch-deutsche Volksbücher I. 2. Aufl. WienHermannstadt 1885 .

Müller-Lisowski IrVM.: Käte Müller-Lisowski, Irische Märchen $=M d W$. Jena, Diederichs.

Musäus: J. K. A. Musäus, Volksmärchen der Deutschen. I-V. Gotha I 782-1786.

Mussäus Mecklenburg: J. Mussäus, Mecklenburgische Volksmärchen. In: Jahrbücher des Vereins für mecklenburgische Geschichte $\mathrm{V}\left(\mathrm{r} 8 \mathbf{4}^{\circ}\right)$.

Naumann IslVM.: Hans und Ida Naumann, Isländische Volksmärchen $=M d W$. Jena, Diederichs.

$N d Z V_{k}$.: Niederdeutsche Zeitschrift für Volkskunde.

Ns.: Niedersachsen. Bremen $1895 \mathrm{ff}$.

Olrik Ragnarök: Axel Olrik, Ragnarök. Die Sagen vom Weltuntergang. Ubertragen von Wilhelm Ranisch. Berlin u. Leipzig 1922.

Panzer Beowulf: F. Panzer, Beowulf. I909. Panzer Hilde-Gudrun: F. Panzer, HildeGudrun. Halle rgor.

Panzer $M S D$.: F. Panzer, Märchen, Sage und Dichtung. München 1905 .

Panzer Urgestalt: F. Panzer, Die Kinder- nud Hausmärchen der Brüder Grimm in ihrer Urgestalt. I-II. München I913.

Pauly-Wissowa: Paulys Real-Encyclopädie der classischen Altertumswissenschaft. Neue Bearbeitung. Herausgegeben von G. Wissowa. Stuttgart $1893 \mathrm{ff}$.

Peter: A. Peter, Sagen und Märchen, Bräuche und Volksaberglauben aus ÖsterreichischSchlesien. Troppau 1867.

Peters Lothringen: F. Peters, Märchen aus Lothringen. Straßburg 1888 .

Petsch Schlüsse: R.Petsch, Formelhafte Schlüsse im Volksmärchen. Berlin 1900.

Pfister Hessen: Hermann v. Pfister, Sagen und Aberglauben aus Hessen und Nassau. Marburg 1885.

Philippson Drosselbart: E. Philippson, Der Märchentypus vom König Drosselbart. Greifswald $1923=F F C$. 50 .

Philo vom Walde: J. Reinelt $=$ Philo vom Walde, Schlesien in Sage und Brauch. Berlin 1883 . 
Pieper Literatur: Pieper, Ägyptische Literaturgeschichte. Wildpark 1927.

Piprek Polnische VM.: Piprek, Polnische Volksmärchen.

Pitrè Fiabe: G. Pitrè, Fiabe, novelle e racconti popolari siciliane. I-IV. Palermo 1875 .

Pitrè Otto Fiabe: G. Pitrè, Otto fiabe e novelle siciliane. Bologna 1873 .

Pitrè Usi: G. Pitrè, Usi e costumi, credenze e pregiudizi del popolo siciliano. I-IV. Palermo 1889 .

Plenzat Typen: Karl Plenzat, Die ost- und westpreußischen Märchen und Schwänke nach Typen geordnet. Elbing 1927.

Poestion: A. Poestion, Isländische Märchen. Wien I 884 .

Polites: N. G. Polites, П $\alpha \rho \alpha \delta 6 \sigma \varepsilon เ \varsigma$ I-II. Athen 1904 .

Pröhle Jugend: H. Pröhle, Märchen für die Jugend. Halle I 854 .

Pröhle $K V M$.: H. Pröhle, Kinder- und Volksmärchen. Leipzig I 853 .

Przibilla OSchlesSM.: F. Przibilla, Oberschlesische Sagen und Märchen. Breslau 1913.

Rank Böhmerwald: J. Rank, Aus dem Böhmerwalde. Leipzig r843, Prag r9r7.

Reusch: R. F. Reusch, Sagein des preußischen Samlandes. Königsberg 1838 . 2. Aufl. 1863.

Rev trad pop.: Revue des traditions populaires. Paris $1886 \mathrm{ff}$.

Rhein WB.: Rheinisches Wörterbuch. Bonn, Klopp. Im Erscheinen.

Ricklin Wunscherfüllung: F. Ricklin, Wunscherfüllung und Symbolik im Märchen. Leipzig: Wien r9o8.

Rittershaus: Adelin eRittershaus, Die neuisländischen Volksmärchen. Halle r9o2.

Rivière: J. Rivière, Recueil de contes populaires de la Kabylie du Djurdjura. Paris 1882 .

Roeder AegMärchen: G. Roeder, Altägyptische Märchen $=M d W$. Jena, Diederichs.

Rolland Faune: E. Rolland, La faune populaire de la France. I-XII. Paris $1876-$ I9Io.

Romanov: E. Romanov, Bělorusskij Sbornik. I-VIII. I 886-I90I.

Saintyves Perrault: $\mathrm{R}$. Saintyves, Les Contes de Perrault et les récits parallèles. Leurs origines. Paris 1923.

Samter $V k$.: Ernst Samter, Volkskunde im altsprachlichen Unterricht. I. Homer. Berlin 1923.

Sch ambach-Müller NdsSM.: G. Schambach und W. Müller, Niedersächsische Sagen und Märchen. Göttingen I 855 .

Schiefner Awarische Texte: Mémoires de l'académie de St.-Petersbourg. ${ }^{7}$ XIX, 6. I873.

Schiller SchlesVM.: A. Schiller, Schlesische Volksmärchen. Breslau 1907 .

Schleicher Litauen: A. Schleicher, Litauische Märchen, Sprichworte, Rätsel und Lieder. Weimar 1857 .

Schmidt Griech.: B. Schmidt, Griechische
Märchen, Sagen und Volkslieder. Leipzig 1877 .

Schneller Wälschtirol: C. Schneller, Märchen und Sagen aus Wälschtirol. Innsbruck I 867 .

Schott WalachM.: Arthur u. Albert Schott, Walachische Märchen. Stuttgart 1845 .

Schultz Urform: F. Schultz, Die Märchen der Brüder Grimm in der Urform. 2. Jahresgabe der Frankfurter Bibliophilengesellschaft 1924 .

SchwAVK.: Schweizerisches Archiv für Volkskunde. Iff. Zürich $1898 \mathrm{ff}$.

Sébillot Folklore: P. Sébillot, Folk-lore de France. I-IV. Paris 1904-1907.

Sébillot Haute-Bretagne: P. Sébillot, Contes populaires de la Haute-Bretagne. I-III. Paris $1880-1882$.

Seif a r t Hildesheim: K. Seifart, Sagen, Märchen, Schwänke und Gebräuche aus Hildesheim. I-II. Göttingen $1854-$ I 860 . 3. Aufl. (ohne Anmerkungen). Hildesheim r9r4.

Simrock $D M .:$ K. Simrock, Deutsche Märchen. Stuttgart 1864 .

Singer SchweizM.: S. Singer, Schweizer Märchen. Anfang eines Kommentars. I-II. Bern 1903, 1906.

Siuts Jenseitsmoive: H. Siuts, Jenseitsmotive im deutschen Volksmärchen. Leipzig 191 I $=$ Teutonia XIX.

Sk la rek: E. Sklarek, Ungarische Volksmärchen. I-II. Leipzig r90I-1909.

Spiegel Bayern: K. Spiegel, Märchen aus Bayern. Blätter zur bayrischen Volkskunde III. Würzburg rgr4.

Spie B VM: K. SpieB, Das deutsche Volksmärchen. Leipzig-Berlin I9r7.

St anitzke Danzig: Stanitzke, Heimatmärchen aus Danzig und Pomerellen. Danzig r924.

Stier-Gaal: G. von Gaal, Ungarische Volksmärchen. Ubbersetzt von G. Stier. Pest 1857 .

Strackerjan Oldenburg: Ludwig Strackerjan, Aberglaube und Sagen aus dem Herzogtum Oldenburg. 2. Aufl., hg. von Karl Willoh. I-II. Oldenburg r919.

Stroebe NordVM.: Klara Stroebe, Nordische Volksmärchen. I-II. Jena I9r5 $(=M d W$.)

Sutermeister $K H M$.: O. Sutermeister, Kinder- und Hausmärchen aus der Schweiz. 2. Aufl. 1873

Taubmann NBöhmen: J. A. Taubmann, Märchen und Sagen aus Nordböhmen. Reichenberg 1887 .

Tegethoff Amor und Psyche: E. Tegethoff, Studien zum Märchentypus von Amor und Psyche. = Rheinische Beiträge IV. BonnLeipzig 1922 .

Tegethoff Franz. VM.: E. Tegethoff, Französische Volksmärchen. I. II. Jena 1923.

Thimme Märchen: A. Thimme, Das Märchen = Handbücher zur Volkskunde II. Leipzig r9o9.

Tille Verzeichnis: V. Tille, Verzeichnis der böhmischen Märchen = FFC. 34 . 
Tonnelat Grimm: E. Tonnelat, Les Contes des Frères Grimm. Paris I9I2.

Tuscan fairy tales. London 1867. 1880.

Uhland Schriften: L. Uhland, Schriften zur Geschichte der Dichtung und Sage. I-VIII. Stuttgart $\mathbf{r} 86_{5} \mathrm{ff}$.

$U B .:$ Am Urdhsbrunnen. Iff. Rendsburg I $88 \mathrm{Iff}$.

UQ: Am Urquell. I-VI. Hamburg r 890 ff. $^{2}$ : I-II, Hamburg-Leiden $1897 \mathrm{f}$.

Veckenstedt Wenden: E. Veckenstedt, Wendische Sagen, Märchen und abergläubische Gebräuche. Graz I880.

Veckenstedt Žmaiten: E. Veckenstedt, Mythen, Sagen und Legenden der Zamaiten. I-II. Heidelberg 1883 .

Vernaleken $K H M$.: Th. Vernaleken, Österreichische Kinder- und Hausmärchen. Wien 1864. 2. Aufl. 1892.

Visentini: I. Visentini, Fiabe mantovane. Torino 1879 .

de Vries $F F C$. 73 .

Waldau $B \not ̈ h m M B .:$ A. Waldau, Böhmisches Märchenbuch. Prag 1860 .

Weber Märchen und Schwank. Diss. Kiel 1904.

Wenzig WestslavMSch.: J. Wenzig, Westslavischer Märchenschatz. Leipzig 1857, I 870 .

Wilhelm China: R. Wilhelm, Chinesische Volksmärchen. Jena r914.

Wisser Grotmoder: W. Wisser, Wat Grotmoder vertellt. Ostholsteinische Volksmärchen. I-III. Leipzig 1904; Jena 1909, I905, 1909.

Wisser Volksmund: W. Wisser, Das Märchen im Volksmund. Hamburg 1925 = Quickbornbücher 32 .

Wisser PlattdVM.: W. Wisser, Plattdeutsche Volksmärchen. Jena r914.

Wlislocki Zigeuner: H. v. Wlislocki, Märchen und Sagen der transsilvanischen Zigeuner. Berlin 1886 .

Woeste: F. Woeste, Die Volksüberlieferungen der Grafschaft Mark. Iserlohn 1 848, 1880 .

Wolf Beitr.: $\dot{F}$. Wolf, Beiträge zur spanischen
Volkspoesie. WSBb. XXXI phil.-hist., I8.59.

Wolf DHM: J. W. Wolf, Deutsche Hausmärchen. Göttingen 1831.

Wolf DMS.: J. W. Wolf, Deutsche Märchen und Sagen. Leipzig 1845 .

Wossidlo Meckl.: R. Wossidlo, Mecklenburgische Volksüberlieferungen. I-III. Wismar 1897-1906.

Wossidlo Unnerierdschen: $\mathrm{R}$. Wossidlo, Von den lütten Unnerierdschen. Rostock o. J. Meckelborger Plattdütsch Bökerie 6/7.

Woycicki-Lewestam Polen: Woycicki-Lewestam, Polnische Volkssagen und Märchen.

WSBb.: Sitzungsberichte der Wiener Akademie der Wissenschaften.

Wuttke Volksaberglauben: Adolf Wuttke, Der deutsche Volksaberglaube der Gegenwart. 3. Bearbeitung von E. H. Meyer. Berlin rgoo.

Zachariae $K l S c h r .:$ Theodor Zachariae, Kleine Schriften. Bonn-Leipzig 1900.

Zaunert DonauM.: Paul Zaunert, Märchen aus dem Donaulande. Jena. $1926(=M d W$.).

Zaunert Märchen seit Grimm: P. Zaunert, Deutsche Märchen seit Grimm. Jena r9r2. (= $M d W$.)

ZdA.: Zeitschrift für deutsches Altertum. Iff. Leipzig $1841 \mathrm{ff}$.

ZEthnol.: Zeitschrift für Ethologie. Iff. Berlin I $869 \mathrm{ff}$.

Zingerle KHMSüddeutschland: Ignaz und Joseph Zingerle, Kinder- und Hausmärchen aus Süddeutschland. Regensburg 1854 .

Zingerle KHMTirol: Ignaz und Joseph Zingerle, Kinder- und Hausmärchen aus Tirol. Innsbruck 1852. 3. Aufl. I9II.

Zingerle Sagen: Ignaz Vincenz Zingerle, Sagen, Märchen und Gebräuche aus Tirol. Innsbruck 1859 .

ZöVk.: Zeitschrift für österreichische Volkskunde. Iff. Wien $1895 \mathrm{ff}$.

ZrwVk.: Zeitschrift für rheinisch-westfälische Volkskunde.

$Z V k .:$ Zeitschrift des Vereins für Volkskunde. Iff. Berlin I $89 \mathrm{Iff}$. 